Rebollo-Catalán, A., de los Santos-Martínez, P. y Jiménez-Cortés, R. (2022). Recursos que ayudan a las adolescentés a recuperarse de una experiencia de violencia de género en el noviazgo. Revista de Investigación Educativa, 40(1), 203-218.

DOI: http://dx.doi.org/10.6018/rie.463081

\title{
Recursos que ayudan a las adolescentes a recuperarse de una experiencia de violencia de género en el noviazgo'
}

\section{Resources that help adolescent girls recover from an experience of gender-related dating violence}

\author{
Ángeles Rebollo-Catalán², Patricia de los Santos-Martínez y Rocío Jiménez-Cortés \\ Universidad de Sevilla
}

\begin{abstract}
Resumen
Este trabajo pretende conocer los recursos que han ayudado a chicas adolescentes a superar una relación de maltrato en el noviazgo, así como su papel en el proceso de recuperación. 13 chicas adolescentes narran su experiencia a través de entrevistas autobiográficas, las cuales se analizan mediante análisis interpretativo basado en la teoría fundamentada. Los resultados muestran que la familia (madres, abuelas y hermanas) y amigas son recursos clave para su recuperación emocional, proporcionando apoyo a lo largo de todo el proceso. También se valora positivamente el programa de atención psicológica a víctimas al poder encontrarse con otras chicas que han sufrido violencia, escuchar su experiencia y poder contar la propia. La denuncia es un recurso esencial para el distanciamiento del agresor, siendo clave la labor policial para presentarla. Las aficiones (danza, teatro, deporte, etc.) suponen un recurso personal clave para recuperar la autoestima y restablecer amistades. La formación en género les permite autoafirmarse y protegerse. Se discuten las implicaciones para la prevención y sensibilización en esta materia.
\end{abstract}

\footnotetext{
1 Este trabajo presenta resultados parciales de la tesis doctoral de Patricia de los Santos Martínez, financiada por el Ministerio de Educación, Cultura y Deporte de España (FPU17/05492) y desarrollada en el marco del proyecto de investigación I+D «Desafíos del Yo: Reconstrucción de las Identidades en Situaciones de Desigualdad y Exclusión Social» (PSI2016-80112-P), financiado por el Ministerio de Economía y Competitividad.

2 Correspondencia: Ángeles Rebollo-Catalán, rebollo@us.es. Departamento de Métodos de Investigación y Diagnóstico en Educación. Facultad de Ciencias de la Educación, Universidad de Sevilla. C/ Pirotecnia, s/n, 41013 Sevilla.
} 
Palabras clave: violencia en el noviazgo; violencia de pareja contra las mujeres; adolescentes; supervivientes; prevención.

\begin{abstract}
This paper tries to discover the resources that help adolescent girls overcome an abusive dating relationship and their role in the recovery process. To this end, 13 adolescent girls narrate their experience through autobiographical interviews, which are examined using interpretive analysis based on grounded theory. The results show that family (mothers, grandmothers and sisters) and friends are key support points for their emotional recovery during the whole process. Programs that offer psychological care for teenage survivors of abusive partnerships are also positively valued, because they offer victims the chance of meeting other girls in similar situations and listening to their experience while giving them a chance to tell of their own. Legal complaints are also an essential resource that allow survivors to distance themselves from their abusers, as are actions taken by the police that encourage teens to report episodes of gender violence. Hobbies (dance, theater, sports, etc.) are a key personal resource to regain self-esteem and to reestablish friendships. Gender training allows them to assert and protect themselves. We also discuss the implications of awareness and prevention in these processes.

Keywords: dating violence; intimate partner violence against women; adolescents; survivors; prevention.
\end{abstract}

\title{
Introducción
}

En Europa (FRA, 2015), el 22\% de las mujeres de más de 15 años ha sufrido violencia física y/o sexual, siendo las adolescentes y jóvenes especialmente vulnerables a sufrir la violencia de género. En España, también encontramos que el 19,3\% de mujeres de 16 a 24 años ha sufrido violencia física y/o sexual en el noviazgo y que el $46,1 \%$ ha padecido algún tipo de violencia psicológica, en especial la violencia de control (DGVG, 2020).

Mulford y Blachman-Demner (2013) consideran la violencia de género en el noviazgo adolescente como una serie de comportamientos abusivos de naturaleza psicológica, física y/o sexual que aparecen en parejas o exparejas pre-adolescentes y adolescentes, que son ejercidos del chico hacia la chica como forma de control, poder y sometimiento.

La violencia de género en parejas adolescentes tiene características propias debido a la etapa psico-evolutiva en que se produce; se trata de violencia muy intensa, variada, que aparece muy tempranamente y de forma brusca en la relación y que se produce en ámbito público, lo que afecta muy negativamente a la chica en el plano emocional (mayor vergüenza, disminución de autoestima, etc.) (Fernández-Zurbarán, 2018; Paz-Rodríguez \& Fernández-Zurbarán, 2014). Esto se origina paralelamente a la normalización con que perciben ciertas conductas machistas en las relaciones íntimas (Nardi-Rodríguez et al., 2019; Pacheco-Salazar \& López-Yáñez, 2019), siendo las de control por celos muy aceptadas como pruebas de amor. Ferrer-Pérez y BoschFiol (2013) apuntan que son las creencias en el amor romántico las que dificultan la percepción y reacción de las chicas a la violencia y las hace más vulnerables a 
experimentarla. Según Donoso-Vázquez et al. (2017) las agresiones asociadas a los mitos del amor romántico y al control coercitivo son la expresión de ciber violencia de género más frecuente.

Sin embargo, son escasas las investigaciones centradas en el proceso de recuperación de adolescentes expuestas a violencia de género en el noviazgo (Margolin, 2019; Reynolds \& Shepherd, 2011) y considerando las características propias de la victimización en adolescentes (Paz-Rodríguez \& Fernández-Zurbarán, 2016) podemos prever que el proceso de recuperación también presente singularidades que hacen necesario su estudio. Además, la investigación reciente sobre violencia en el noviazgo adolescente (Malhotra et al., 2015; Storer \& Strohl, 2017) señala la necesidad de atender a los factores de protección para mejorar la prevención. En esta línea, Taylor et al. (2021) propone incorporar relatos y experiencias de adolescentes supervivientes poniendo el énfasis en los factores protectores como vía de mejora de los programas de prevención.

El proceso de recuperación de una relación de violencia de género ha sido definido como el camino que experimenta la mujer desde que rompe definitivamente con su agresor hasta que logra reconstruir su vida personal y social con autonomía y libertad (Roca \& Masip, 2011; Sinko \& Saint-Arnault, 2020). Allen y Wozniak (2014) demuestran que este proceso va más allá de la curación del trauma e implica trabajar en grupo con otras supervivientes a nivel psicológico, espiritual, social y cultural para que logren reconstruir su identidad y restaurar su trayectoria vital. Algunas investigaciones (Reynolds \& Shepherd, 2011) han mostrado que el proceso de recuperación en adolescentes comienza con momentos cruciales de reparación de su identidad a través de la implicación en actividades propias de su edad, la separación de la familia del agresor y la reconstrucción de relaciones familiares. González-Guarda et al. (2016) demuestra que estos eventos específicos (puntos de inflexión) sirven para que las adolescentes tomen conciencia de que necesitan ayuda y comiencen su recuperación.

Cala (2011) identifica una variedad de recursos sociales e individuales que ayudan a las mujeres en el proceso de recuperación, encontrando que la fuerza interior y una actitud proactiva junto con la confianza en la utilidad de los recursos que tienen a su alcance resultan clave en este proceso. Fleming \& Resick (2017) demuestran que las creencias en la utilidad de los recursos son un potente factor que influye en la conducta activa de búsqueda de ayuda. Flasch et al. (2017) también constata una variedad de recursos que favorecen la recuperación, entre los que señala la formación en violencia de género para detectar relaciones abusivas y usar las propias experiencias para ayudar a otras.

Por su parte, Rebollo-Catalán et al. (2010), identifican el papel clave que juegan ciertas emociones en el proceso de recuperación, identificando el optimismo en la propia capacidad para cambiar su vida, el alivio que supone la soledad como espacio de crecimiento personal, el orgullo por su valía personal y el miedo que las hace estar alertas y ser cautas en el proceso de ruptura y separación. Donoso-Vázquez (2020) encuentra que en el proceso de superar las secuelas de la violencia, se hace necesario superar el miedo, aumentar la autoestima y eliminar sentimientos de culpa. Ruiz-Pérez et al. (2013) destacan el sentimiento de protección de su entorno íntimo como desencadenante de la ruptura y de la búsqueda de ayuda. 
Otras investigaciones han demostrado la importancia del apoyo social en el proceso de recuperación (Choi et al, 2019; Ruiz-Repullo, 2016; Zapor et al., 2018). RebolloCatalán y Mayor-Buzón (2020) encuentran que las adolescentes acuden sobre todo a la familia y grupo de iguales para pedir ayuda. Según Paz-Rodríguez y FernándezZurbarán (2014), el grupo de iguales juega un papel muy importante en esta etapa pudiendo convertirse en un recurso para la detección temprana de la violencia y el apoyo a la chica que la sufre. La investigación (González-Guarda, 2016; Sabina \& Ho, 2014) ha demostrado que las adolescentes buscan más ayuda informal de amigos y familiares que de fuentes formales de servicios profesionales. Este apoyo informal del círculo íntimo es clave para que accedan a recursos institucionales de carácter legal, médico y policial (Evans \& Feder, 2016; Hedge et al., 2017), más aún entre adolescentes que muestran un gran desconocimiento de estos recursos institucionales (Rebollo-Catalán et al., 2018; Rebollo-Catalán \& Mayor-Buzón, 2020), entre los cuales se encuentra la atención psicológica especializada. Santos et al. (2017) demuestran el impacto positivo de la intervención psicológica en grupo, mejorando no solo síntomas clínicos (depresión, ansiedad, etc.) sino también su autoestima, su apoyo social y su comprensión de la violencia.

\section{Planteamiento y objetivos}

El objetivo general es conocer los recursos que ayudan a las adolescentes víctimas de violencia de género en su recuperación de una relación de maltrato en el noviazgo, indagando en el papel que juegan en el proceso de recuperación. Esto permitirá mejorar la atención a las víctimas y aportar un conocimiento valioso para la sensibilización y prevención de la violencia de género.

Queremos identificar los recursos que más han ayudado a las chicas que han sobrevivido a una experiencia de maltrato en el noviazgo narrada en su propia voz e indagar en el papel que desempeñan en su propio proceso de recuperación. Por ello, ponemos el acento no solo en su identificación sino también en la valoración y reflexión que hacen del papel que han jugado en su proceso de recuperación.

\section{Método}

Desarrollamos una investigación biográfica-narrativo mediante un diseño de relatos paralelos, aplicando para su realización las recomendaciones metodológicas de la investigación feminista (Woodiwiss et al., 2017) y las recomendaciones éticas de la Organización Mundial de la Salud (2016) para la investigación en violencia de género.

\section{Participantes}

Participan en el estudio 13 mujeres supervivientes (tabla 1) de una relación de violencia de género durante el noviazgo en la adolescencia, con edades actuales comprendidas entre los 17 y los 25 años. 
Tabla1

Perfil de las participantes

\begin{tabular}{lcccc}
\hline \multirow{2}{*}{ Participantes } & Edad actual & Duración relación & \multicolumn{2}{c}{ Edad inicio de la relación } \\
\cline { 3 - 5 } & & & Superviviente & Agresor \\
\hline P1 & 20 & 2 & 13 & 16 \\
\hline P2 & 17 & 1 & 15 & 18 \\
\hline P3 & 19 & 1 & 14 & 17 \\
\hline P4 & 25 & 3 & 20 & 20 \\
\hline P5 & 19 & 3 & 12 & 13 \\
\hline P6 & 18 & 1 & 14 & 14 \\
\hline P7 & 22 & 1 & 15 & 15 \\
\hline P8 & 20 & 2 & 16 & 23 \\
\hline P9 & 25 & 8 & 16 & 16 \\
\hline P10 & 19 & 3 & 15 & 16 \\
\hline P11 & 21 & 3 & 15 & 15 \\
\hline P12 & 19 & 5 & 12 & 16 \\
\hline P13 & 19 & 1 & 13 & \\
\hline
\end{tabular}

Para su selección hemos aplicado un muestreo no probabilístico utilizando una combinación de muestreo de casos típicos y en bola de nieve, considerando como criterios de inclusión: a) que la chica hubiera puesto fin a la relación, b) que hubiera tomado distancia con el agresor y su entorno y, c) que hubiera recuperado el control sobre su vida personal, social y familiar (Cala, 2011; Roca \& Masip, 2011).

\section{Instrumento}

Empleamos una entrevista semiestructurada autobiográfica para recoger las experiencias de las chicas narradas en su propia voz y aproximarnos a las circunstancias que dan sentido a sus experiencias. Aunque el guión incluía siete bloques temáticos en los que se preguntaba por el inicio de la relación y los primeros episodios de maltrato, pasando por la ruptura y la recuperación hasta su situación actual, expectativas de futuro y recomendaciones para otras chicas, en el presente trabajo nos hemos centrado exclusivamente en las preguntas sobre ruptura, recuperación y situación actual. La aplicación del guión en cada encuentro se ha hecho de forma flexible y abierta partiendo de la situación, contexto y espontaneidad de la chica.

\section{Procedimiento}

Para la recogida de información contamos con la colaboración del Instituto Andaluz de la Mujer y la Unidad para la Igualdad de la Universidad de Sevilla.

Las terapeutas del Programa de Atención Psicológica a Adolescentes Víctimas de Violencia de Género de Andalucía, presentaron el estudio a las chicas y sus familias 
y recopilaron los consentimientos informados firmados por las participantes y sus tutores/as legales y acordaron las fechas de realización de las entrevistas.

Se localizó y accedió también a algunas participantes a través de informantes clave en acciones formativas organizadas por la Unidad para la Igualdad de la Universidad de Sevilla en materia de violencia de género, a las que se presentó el estudio y se consultó su disponibilidad para participar en el mismo.

Todas las entrevistas fueron realizadas por tres investigadoras con formación en violencia de género en un horario y lugar adaptado a las necesidades de las chicas según su disponibilidad. Los espacios en los que realizamos la entrevista fueron el centro provincial donde las chicas habían asistido a terapia o en sus centros de estudios, en los que se sentían a gusto y seguras para expresarse libremente lo que facilitaba un clima de confianza y ambiente sin interrupciones.

Las entrevistas fueron grabadas en audio y posteriormente transcritas eliminando datos de identificación o localización, utilizando pseudónimos y suprimiendo referencias del contexto. Además, se comunicó a las participantes la posibilidad de interrumpir su participación si así lo deseaban.

\section{Análisis de datos}

Hemos aplicado un análisis interpretativo de carácter inductivo basado en la teoría fundamentada (Strauss \& Corbin, 1998) para indagar en los significados y acciones de las participantes y las condiciones en las que éstas emergen. Para ello, y aunque partíamos de dos conceptos previamente definidos en la investigación (recursos y momentos), su desglose y operativización en categorías ha seguido un método inductivo.

Nuestro análisis comenzó leyendo y tomando notas detalladas sobre las entrevistas para identificar de forma precisa y aplicada ambos conceptos en nuestros datos. En esta fase identificamos 556 unidades de análisis, llegando al acuerdo de que un recurso constituía cualquier elemento intrínseco o extrínseco a la chica que desencadenaba la ruptura de la relación y su posterior recuperación, siendo clave la referencia a voces, mensajes, pensamientos, acciones, gestos y emociones que supusieran un apoyo para ganar autonomía y reconstruir su vida. Para contextualizar el papel que desempeñaba cada recurso en el proceso de recuperación, se consideró su desglose en momentos cruciales, los cuales representaban eventos, situaciones o sucesos que les servían para tomar conciencia, encaminarse a romper la relación y asumir las riendas de su vida.

Una vez realizada esta exploración inicial, elegimos dos entrevistas para desarrollar una lista de categorías que aplicamos de manera independiente dos investigadoras, llevando a cabo dos rondas de codificación hasta alcanzar un consenso del $80 \%$, enfoque validado empíricamente (Creswell \& Poth, 2018). En esta fase utilizamos anotaciones que nos permitieran mediante el método de comparación constante sistematizar la codificación; también aplicamos el método de comprobación cruzada, anotando las condiciones en que se daba cada recurso para un análisis más profundo.

Posteriormente, se aplicó el sistema de categorías de forma extensiva y sistemática a todas las entrevistas, refinando, ampliando y precisando aún más dicho sistema. En esta fase, las investigadoras mantuvieron diversas reuniones para debatir y consensuar la ampliación, revisión o actualización del sistema inicial de categorías, volviendo a 
las transcripciones de las entrevistas y a la memoria de anotaciones de cada entrevista para asegurar que el análisis mantuviera el sentido otorgado por la chica a su experiencia. Fruto de este proceso, la categoría recursos se aplicó a un total de 463 extractos, desglosándose en tres subcategorías: a) recursos sociales, referidos a la red social de apoyo de la adolescente; b) recursos individuales, referidos a las cualidades, actitudes, pensamientos y emociones que las ayudan $\mathrm{y}, \mathrm{c}$ ) recursos institucionales, referidos a la red de profesionales que desde ámbito institucional apoyan. La categoría momentos se aplicó 284 extractos, desglosándose en diez subcategorías: a) rompiendo la relación con el agresor; b) tomando distancia del agresor y su entorno; c) reparando relaciones familiares; d) recibiendo terapia psicológica; e) recuperando actividades; f) reconstruyendo lazos de amistad; g) asumiendo su autocuidado y bienestar; h) comenzando nuevas relaciones de pareja.

Para el análisis interpretativo y extracción de resultados, partimos de tablas cruzadas de frecuencia de ambas categorías para valorar la participación de los recursos en cada momento del proceso de recuperación, pero esto ha sido solo el punto de partida para la interpretación, ya que nos centramos sobre todo en el significado atribuido por las participantes, buscando lo común en la experiencia vivida por ellas. Por último, hemos realizado una comprobación con las participantes para asegurar que los resultados y su interpretación reflejan fielmente su experiencia. Esta consulta ha servido para enriquecer la interpretación y posterior discusión de los resultados y sus implicaciones educativas.

\section{Resultados}

Los resultados muestran que el círculo social íntimo de las adolescentes (40.4\%) supone una fuente fundamental de ayuda para su recuperación, siendo la familia $(f=103)$ el recurso más presente a lo largo de todo el proceso de recuperación. Este apoyo tiene un marcado carácter femenino, ya que son madres $(f=53)$, abuelas $(f=$ 13), primas $(f=9)$ y hermanas $(f=7)$ quienes más contribuyen en su recuperación, quienes más contribuyen en su recuperación, siendo clave su intervención en la toma de distancia con el agresor y en los momentos de soledad y bajón emocional tras la ruptura y durante el proceso judicial.

Ese apoyo se materializa en sentirse acompañadas con acciones y gestos que les hacen saber que están de su lado, sirviendo para reparar vínculos familiares.

Mi madre pasó de estar histérica a dejarme llorar (...) y eso fue lo mejor, que mi madre viniese y me dijese "tiempo al tiempo que pasa, esto pasa" y yo siempre pensaba "esto pasa, pasa" (P6).

Cuando me apretaba el pecho y me costaba respirar, me ponía muy nerviosa, mi abuela se ponía conmigo "tú tranquila" me daba una bolsa y me decía "¿quieres que nos vayamos a urgencias?" (P1).

Pero también se manifiesta a nivel emocional en reforzar la autoestima con mensajes positivos. 
Mi hermana me ponía todas las mañanas delante del espejo y me decía que repitiese que era bonita, que valía y entonces pues eso lo hacía todas las mañanas, era una forma de recalcarme la autoestima, y cuando flaqueaba eso era lo que yo... (P12).

También las amigas $(f=59)$ son un apoyo clave en el proceso de recuperación, al llenar el vacío con actividades y planes que le hacen estar activas, escuchándolas activamente y apoyándolas emocionalmente.

Julia siempre ha estado conmigo, ella me animaba "vamos Elena vamos a apuntarnos en este taller, vamos a tal sitio" "mira, están echando esto en la televisión" y ella fue la que siempre estuvo (P4).

Mis amigas lo hacían todo con normalidad, me trataban con normalidad (chasqueo de lengua) como si nada hubiese pasado (P13).

Este círculo íntimo formado por madres, abuelas, hermanas y amigas les ayudan también para acudir a los recursos institucionales a pedir ayuda y fortalecer su decisión.

Yo no quería venir (a terapia) porque lo pasaba mal y le decía a mi madre es que cada vez que salgo, salgo llorando y yo no quiero ir y me decía sales llorando porque lo que hace es moverte, tienes que ir para eso, para sacarlo y me decía lo que llores hoy, ya no lo lloras mañana (P6).

En algunos casos, ellas recalcan la formación y experiencia de las personas de su entorno íntimo como algo clave para que su ayuda resulte efectiva.

Hablando con mi prima, que es muy buena psicóloga y me fue abriendo los ojos, pero fue sentir que no estaba sola, que mi prima iba a estar conmigo y podía sentir su ayuda (P9).

Yo tenía a Julia, una amiga que había sufrido violencia de género, que siempre estaba al lado mía, le contaba todo y estuvo conmigo intentando hacerme ver las cosas (P4).

Los resultados también nos muestran que los recursos institucionales $(16 \%)$ tienen un papel decisivo en la recuperación, pero estos se movilizan sobre todo en la ruptura y toma de distancia del agresor, que constituyen momentos de mayor riesgo y vulnerabilidad de las chicas. Ellas reconocen la ruptura como un proceso con recaídas y retrocesos, donde la policía $(f=8)$ influye de forma determinante para interponer la denuncia $(f=11)$ y las posteriores medidas judiciales de distanciamiento del agresor, lo que se produce cuando la escalada de violencia aumenta y se hace tan abrumadoramente explícita que no la pueden ocultar.

Quien me abrió los ojos fue la policía que me dijeron "que denunciamos nosotros, que la denuncia ya va a estar puesta" y me dijeron "¿vas a dejar que vuelva otra 
vez a tu vida?" que me dijera eso la policía me influyó mucho (...) la policía fue mi gran bote salvavidas, los que me hicieron abrir los ojos y me dieron fuerza (P1).

Ellas confiesan que poner distancia les proporciona un respiro importante para poder reconectar con ellas mismas y, en este sentido, más allá de herramientas como la orden de alejamiento o la prisión, que ellas valoran positivamente, de nuevo familia y amigas proporcionan el refugio necesario para protegerse y aislarse de la omnipresencia del agresor y de su entorno, siendo importante su complicidad para cortar toda comunicación.

Cuando estaba con ellas (sus amigas), me estaba llamando al móvil y ellas me lo bloquearon de todos lados, las llamadas, todo, todo, más contenta que la mar, liberada completamente, ya me lo había quitado de encima para siempre (P11).

Lo dejé:: y me fui a casa de mi hermana un par de semanas, para quitarme de aquí y:: u::mm como que me desintoxiqué un poco de él y yo vi que yo no tenía que aguantar eso, allí con mis cavilaciones, como desintoxiqué (P8).

Entre los recursos institucionales destaca la terapia psicológica $(f=40)$ que la mayoría recibe a través del programa de atención a menores víctimas de violencia de género y, que supone un punto de inflexión en su proceso de recuperación puesto que las ayuda a reencontrarse con ellas mismas y las impulsa a tomar las riendas de su vida. Además de superar el trauma y recuperar la autoestima, la terapia les descubre un entorno amigable libre de juicios donde expresar su experiencia y sentirse acogidas.

Más que nada era para reforzar mi autoestima porque cuando yo lo dejé, acabé por los suelos, estaba destrozadita y me ayudó mucho la terapia de autoestima, de empoderamiento (P3).

Es mi zona de confort, todas nos sentimos a gusto, nos ayudamos unas a otras, nos desahogamos, sabes que no te van a juzgar, las tienes al lado porque están pasando lo mismo (P12).

Pero también supone un entorno para autoconocerse y aprender sobre el amor y sobre relaciones amorosas.

También te dan mucha educación sobre las relaciones tóxicas y estoy bien informada pues cada día que vengo aprendo algo nuevo, hablamos de libros y me ayuda mucho aprender cosas nuevas sobre estos temas, te hace abrir mucho los ojos (P2).

Yo venía con dolor de cabeza pero después me venía superbién, me ha aportado aprender cómo es de verdad tener una pareja que:: esté pendiente de ti, que te quiera, es todo lo que me han enseñado (P12). 
Este aprendizaje que genera el proceso terapéutico les lleva a descubrir nuevos espacios y actividades que les ayudan a fortalecerse y convertir su experiencia en algo positivo que ayude a otras chicas.

Conocer el feminismo me ayudó a entender muchas cosas de mí, no sé cómo llegué, a lo mejor por entrar aquí, pero a partir de ahí, empecé:: a interesarme más y te ves vídeos, lees cosas, hablas con gente que también está en ese ambiente (P7).

Porque viví esto empecé a meterme en el tema feminismo, en violencia de género y todo esto y fue como darme cuenta de todo lo que había pasado, fue como un cambio, o sea, yo creo que hay dos Lauras, la de antes y la de después (P5).

En cuanto a los recursos individuales (43.6\%), los relatos muestran la gran variedad de recursos que movilizan las chicas para recuperarse, aumentando su uso con el transcurso del proceso de recuperación. La autonomía o capacidad para conducir su vida de forma independiente $(f=30)$ es el más presente en todos los relatos pero éste aparece muy avanzado el proceso y tras la intervención terapéutica, que les ayuda a reivindicar el espacio personal propio como parte de su nuevo yo, aprendiendo a estar solas y valorando y protegiendo su tiempo para escucharse y descubrirse.

Me he dado cuenta de que estoy tan bien sola, que no me hace falta nadie, que para cuidarme ya estoy yo (P2).

Me he dado cuenta de que los espacios personales son fundamentales, ya no estoy diariamente con una persona (P6).

Dos recursos personales son necesarios para que se produzca la ruptura definitiva; por un lado, el miedo protector $(f=19)$, no tanto de su vida, como de las personas de su entorno (hermanos, abuelas, amigos) y, por otro el desencanto $(f=23)$ o dejar de creer en la relación y en el agresor; es ese cansancio y agotamiento al ver que no podrán tener la relación que desean y comprender que él no es la persona que ellas creían lo que las hace desprenderse.

Yo me sentía muy enclaustrada, teníamos muchas discusiones, lo intenté dejar varias veces porque digo ¿si tan mala novia soy por qué sigues conmigo? Ya está, digo, lo dejamos porque yo ya no puedo más (P7).

La que está contigo soy yo, pero nadie más, y a mí que me toques algo mío, que de verdad es mío, no tuyo, a mi eso ya::: me tuvo::.:. fue lo que me hizo decir "no, ya está" (P6).

Esta decisión de ruptura trae consigo un sentimiento de alivio $(f=8)$, al librarse del peso que supone ya esta relación en sus vidas, ayudándolas a desvincularse emocionalmente del agresor. 
Me sentí como si me hubiesen quitado la cadena de los pies, de los presos, pues igual; liberada, me entró una felicidad por dentro y empecé ya a decir, voy a disfrutar (P4).

Sentía que me había quitado un problema de encima, como si me hubiese quitado, kilos, kilos y kilos de encima, pues así me sentía, muy feliz, libre, contenta conmigo misma (P11).

En la toma de distancia con el agresor, resulta crucial mantenerse activas, lo que ellas canalizan a través de aficiones, intereses y actividades o proyectos personales abandonados durante la relación $(f=23)$ que junto con mantener una actitud positiva $(f=12)$, determinación $(f=9)$ y fortaleza $(f=6)$ le ayudan a mantener la decisión. Todas expresan su firmeza para mantener su decisión y su espíritu de superación.

Le bloqueé de todos lados y ... bueno, él después intentando ¿no? pero yo tenía claro que no, cuando tomé esa decisión, te lo digo de verdad, me costó un montón de trabajo tomarla, pero cuando la tomé, desde ese día digo: "se acabó" y así fue; yo tenía claro que lo tenía que superar y no podía quedarme estancada (P3).

Las aficiones y hobbies refuerzan su autoestima porque les sirve para hacer algo que se les da bien y les gusta (pintura, música, deporte, teatro, etc.), siendo en la práctica de estas actividades donde reconectan con otras personas y les da la oportunidad de formar nuevos vínculos de amistad.

Sentía que no pintaba nada aquí, pero entonces me vi unas canastas, yo jugaba al baloncesto, me compré una pelota y empecé a jugar, de primeras yo sola, pero empecé a conocer gente con mis mismas necesidades y fui abriéndome (P1).

Los estudios y fijarse metas a corto y medio plazo supone una fuente de autorrealización, que las hace sentirse orgullosas y empoderadas $(f=12)$.

Me gradué y dije "yo puedo", después me metí en un grado medio y digo "es que yo puedo, es que valgo", ese sentimiento de que no vales se te queda, esa sensación de pérdida al haber apostado tanto por esa relación, así que retomarlo, me dio mucha fortaleza (P1).

Yo me refugiaba en los estudios, entonces le daba al botón de off de todo y me centrada nada más en los estudios, con los estudios era yo me sentía poderosa, veía los logros que yo conseguía (P4).

La formación en violencia $(f=20)$, el autoconocimiento $(f=16)$ y la cautela $(f=16)$ resultan cruciales en el momento de comenzar nuevas relaciones de pareja, lo que las lleva a encaminarlas de forma realista y calmada, expresando sus necesidades y estableciendo sus ritmos. 
Yo confío en él y él en mí, es una relación totalmente diferente, pero también porque me he formado yo, porque si tú tienes una actitud de mejorar, de informarte, apuntarte a cosas, entonces ahora la relación es diferente, soy una naranjita enterita y no pienso que sin él me vaya a morir (P3).

Ahora voy un poco u::m:, digamos, que con pies de plomo en el sentido de que no me creo nada de lo que dicen. Me pueden decir te quiero, te adoro, te llevo a los toros (P9).

En este sentido, debemos mencionar el apoyo que supone la nueva pareja $(f=19)$ para volver a creer en el amor y recomponer sus heridas en un plano íntimo, siendo lo que más les cuesta recomponer.

Fue sobre todo sorpresa, por así decirlo, fue como inesperado, para mí que yo le dijera a mi pareja: "oye mira, voy a quedar más tarde con mis amigas, vete tú" y que él me dijera: "vale, pues nos vemos mañana" para mí eso era como: "ino se ha enfadado!, ¿Qué fuerte!" y entonces fue sorpresa y de aprender y de empezar a valorarme (P5).

Nuestro análisis también revela las escasas intervenciones de profesorado $(f=6)$, vecinos $(f=4)$ o familia del agresor $(f=2)$, que ayuden realmente a las chicas en su recuperación. Estas intervenciones son muy puntuales y se limitan exclusivamente a detener episodios de violencia física extrema.

\section{Discusión y conclusiones}

El propósito de este estudio es descubrir los recursos que ayudan a chicas adolescentes en su proceso de recuperación de una experiencia de violencia de género en el noviazgo.

Los resultados muestran la gran variedad de recursos sociales, individuales e institucionales que se movilizan en dicho proceso, siendo más abundantes y decisivos en la ruptura y toma de distancia del agresor. Esto concuerda con los resultados de investigaciones previas (Cala, 2011; Ruiz-Repullo, 2016) que muestran también la diversidad de recursos que ayudan en el proceso de recuperación.

De este modo, como desencadenantes de la ruptura encontramos que el miedo, el desencanto y la policía entran en juego cuando la violencia aumenta, se hace más frecuente e intensa y las amenazas alcanzan al círculo íntimo. El miedo por proteger a su familia y amigos supone un acicate para la ruptura. Esto concuerda con los resultados de investigaciones en adultas (Ruiz-Pérez et al., 2013). No obstante, la ruptura se produce cuando dejan de idealizar la relación y de creerlo a él, es lo que ellas expresan como cansancio, agotamiento y desencanto al comprender que no es la relación que ellas querían y creían tener. Desprenderse de las creencias en el amor romántico resulta clave para la ruptura definitiva. Ferrer-Pérez y Bosch-Fiol (2013) establecen una relación entre estas creencias y la violencia de género, considerándolas dañinas para el establecimiento de relaciones amorosas sanas. En este contexto, ellas valoran positivamente la intervención policial para interponer la denuncia, lo que favorece la activación de otros recursos.

El distanciamiento del agresor y de su entorno se revela un momento crucial para la recuperación, en el que actúan recursos determinantes. En primer lugar, la familia a 
través de madres, abuelas, primas y hermanas que refuerzan la autoestima de la chica con mensajes positivos sobre su valía y cuyas voces van desplazando poco a poco la del agresor. Esto concuerda con hallazgos de otras investigaciones (González-Guarda, 2016; Sabina et al., 2014; Zapor et al., 2018) que han mostrado el apoyo social como factor facilitador de la recuperación. Pero también las amigas resultan clave para llenar el vacío tras la ruptura, siendo cómplices necesarias para bloquear los intentos de contacto del agresor y de su entorno con ellas.

Familia y amigas también las ayudan a acudir a recursos institucionales y, en especial, a terapia psicológica, que les servirá para superar el trauma y recuperar su autoestima, pero también para autoconocerse y tomar las riendas de su vida, llevándolas a valorar la autonomía y los espacios personales como anclaje de su nuevo yo. De la terapia, ellas destacan las sesiones en grupo con otras chicas para desahogarse y hablar de su experiencia en un entorno libre de juicios, lo que supone un gran alivio. Santos et al., (2017) también demostraron el impacto positivo de la intervención psicológica en grupo para recuperarse de la violencia lejos del estigma de la etiqueta de víctima. La terapia también les proporciona un recurso al que dan mucho valor: la formación feminista y el conocimiento de la violencia de género, que les ayuda a conducir sus nuevas relaciones de pareja. Flasch et al., (2017) ya identificaron la importancia de la formación como factor protector en mujeres adultas víctimas de violencia de género.

Nuestro estudio también descubre la importancia de las aficiones y otras actividades en la recuperación de la autoestima y en su progresiva autonomía. Aunque Reynolds y Shepherd (2011) mostraron la implicación en actividades propias de su edad como factor clave en la recuperación, nuestro estudio va más allá. Por un lado, encontramos que las aficiones sirven para fortalecer la autoestima, llenar el vacío que deja la ruptura y facilitar nuevos vínculos de amistad y, por otro, proyectos y actividades abandonadas, entre los que destacan los estudios, les sirve para recuperar su agencia perdida y suponen una vía de empoderamiento personal (Donoso-Vázquez, 2020; Martin et al., 2019).

Nuestro estudio aporta evidencias para la mejora de los programas de prevención y sensibilización en violencia de género en la adolescencia, documentando los factores protectores que resultan cruciales en la recuperación. En concreto, el estudio revela la importancia de fortalecer la autoestima a través de las aficiones y otras actividades para aumentar la agencia, fomentar la autonomía y los espacios personales y entrenar en relaciones amorosas sanas. El estudio también revela el papel crucial que desempeña la formación en violencia de género de personas del entorno (familia, vecindario, amistades, profesorado) para que su ayuda sea efectiva, revelándose las actividades y espacios feministas una valiosa vía de obtención de ese conocimiento.

\section{Agradecimientos}

Queremos agradecer a las participantes y sus familias su aceptación para participar en el estudio y la generosidad con la que nos han compartido sus experiencias y a las personas responsables y equipo de profesionales vinculado al Instituto Andaluz de la Mujer y a la Unidad para la Igualdad de la Universidad de Sevilla por su inestimable ayuda para la realización del estudio. A todas queremos expresar nuestro reconocimiento por su labor, compromiso y lucha para erradicar la violencia de género. 


\section{Referencias}

Allen, K.N., \& Wozniak, D. F. (2014). The Integration of Healing Rituals in Group Treatment for Women Survivors of Domestic Violence. Social Work in Mental Health, 12(1), 52-68. https://doi.org/10.1080/15332985.2013.817369

Cala, M. J. (Ed.). (2011). Recuperando el control de nuestras vidas: reconstrucción de identidades y empoderamiento en mujeres víctimas de violencia de género. Instituto de la Mujer del Ministerio de Sanidad. https://bit.ly/2W77fty

Choi, A. W., Liu, L. L., Chan, P. Y., Lo, R. T., Wong, J. Y., \& Tang, D. H. (2019). The Significance of Volunteer Support for Female Survivors Recovering from IntimatePartner Violence. The British Journal of Social Work, 49(7), 1778-1797. https://doi. org/10.1093/bjsw/bcy109

Creswell, J. W., \& Poth, C. N. (2018). Qualitative inquiry and research design: Choosing among five approaches. Sage Publications.

Delegación del Gobierno contra la Violencia de Género (DGVG) (2020). Macroencuesta de violencia contra la mujer 2019. Ministerio de Igualdad. https://bit.ly/379RYyh

Donoso-Vázquez, T., Rubio-Hurtado \& Vilá-Baños, R. (2017). Las ciberagresiones en función del género. Revista de Investigación Educativa, 35(1), 197-214. http://dx.doi. org/10.6018/rie.35.1.249771

Donoso-Vázquez, T. (2020). Superar las secuelas de la violencia machista. Las voces de las mujeres y sus hijas e hijos. Egregius.

European Union Agency for Fundamental Rights (FRA) (2015).Violence against women: An EU-wide survey. Main results. Publications Office of the European Union. https:// doi.org/10.2811/981927

Evans, M.A. \& Feder, G.S. (2016). Help-seeking amongst women survivors of domestic violence: A qualitative study of pathways towards formal and informal support. Health Expectation, 19(1), 62-73. https://doi.org/10.1111/hex.12330

Fernández-Zurbarán, P. (2018). Guía para profesionales ante chicas adolescentes que sufren violencia de género: saber mirar, saber acoger, saber acompañar. Instituto Andaluz de la Mujer, Consejería de Igualdad y Políticas Sociales de la Junta de Andalucía. https:// bit.ly/36oLHhZ

Ferrer-Pérez, V. \& Bosch-Fiol, E. (2013). Del amor romántico a la violencia de género. Para una coeducación emocional en la agenda educativa. Profesorado: Revista de currículum y formación del profesorado, 17(1), 105-122. https://www.ugr.es/ recfpro/ rev171ART7.pdf

Flasch, P., Murray, C.E. \& Crowe, A. (2017). Overcoming Abuse: A Phenomenological Investigation of the Journey to Recovery from Past Intimate Partner Violence. Journal of Interpersonal Violence, 32(22), 3373-3401. https://doi.org/10.1177/0886260515599161

Fleming, C.J. \& Resick, P.A. (2017). Help-Seeking Behavior in Survivors of Intimate Partner Violence: Toward an Integrated Behavioral Model of Individual Factors. Violence and Victims, 32(2), 195-209. https://doi.org/10.1891/0886-6708.VV-D-15-00065

González-Guarda, R.M., Ferranti, D., Halstead, V. \& Ilias, V.M. (2016). Experiences with Dating Violence and Help Seeking Among Hispanic Females in Their Late Adolescence. Issues in Mental Health Nursing, 37(4), 229-238. https://doi.org/10.310 9/01612840.2016.1141341 
Hedge, J.M., Sianko, N. \& McDonell, J.R. (2017). Professional Help-Seeking for Adolescent Dating Violence in the Rural South: The Role of Social Support and Informal Help-Seeking. Violence Against Women, 23(12), 1442-1461. https://doi. org/10.1177/1077801216662342

Malhotra, K., González-Guarda, R.M. \& Mitchell, E.M. (2015). A Review of Teen Dating Violence Prevention Research: What About Hispanic Youth? Trauma, Violence and Abuse, 16(4), 444-465. https:/doi.org/10.1177/1524838014537903

Margolin, I. (2019). Breaking Free: One Adolescent Woman's Recovery from Dating Violence Through Creative Dance. American Journal of Dance Therapy, 41(2), 170-192. https:/doi.org/10.1007/s10465-019-09311-9

Martin, S.L., McLean, J., Broocks, C. \& Wood, K. (2019). “I've Been Silenced for so Long": Relational Engagement and Empowerment in a Digital Storytelling Project With Young Women Exposed to Dating Violence. International Journal of Qualitative Methods, 18, 1-12. https:/doi.org/10.1177/1609406919825932

Mulford, C.F. \& Blachman-Demner, D.R. (2013). Teen Dating Violence: Building a Research Program Through Collaborative Insights. Violence Against Women, 19(6), 756-770. https://doi.org/10.1177/1077801213494705

Nardi-Rodríguez, A., Pastor-Mira, M.A., López-Roig, S. \& Ferrer-Pérez, V.A. (2019). What do adolescents Believe About Performing and Accepting Intimate Partner Violence Behaviors? A Reasoned Action Approach. Journal of Family Violence, 34(5), 461-477. https:/doi.org/10.1007/s10896-019-00060-4

Organización Mundial de la Salud (2016). Ethical and safety recommendations for intervention research on violence against women. RTI International. https://bit.ly/3gBHkUl

Ozge, A. (2020). A feminist approach to dating violence prevention: Creating change towards safety, equality and mutuality. Feminism \& Psychology, 30(2), 143-164. https:/ doi.org/10.1177/0959353519882462

Pacheco-Salazar, B. \& López-Yáñez, J. (2019). “Ella lo provocó": el enfoque de género en la comprensión de la violencia escolar. Revista de Investigación Educativa, 37(2), 363-378. https://doi.org/10.6018/rie.37.2.321371

Paz-Rodríguez, J.I. \& Fernández-Zurbarán, P. (2014). Guía para madres y padres con hijas adolescentes que sufren violencia de género. Instituto Andaluz de la Mujer. Conserjería de Igualdad, Salud y Políticas Sociales de la Junta de Andalucía. https://bit.ly/342ZMQB

Paz-Rodríguez, J.I. \& Fernández-Zurbarán, P. (2016). En el laberinto... Manual de uso de la campaña \#No te pierdas sin libertad no hay amor. Instituto Andaluz de la Mujer. Conserjería de Igualdad, Salud y Políticas Sociales de la Junta de Andalucía. https:// bit.ly/3qNiFD5

Rebollo-Catalán, A., Cala Carrillo, M.J, Vega Caro, L., Godoy Hurtado, M. S. \& Sabuco i Cantó, A. (2010).Metáforas emocionales en el proceso de recuperación de mujeres víctimas de violencia de género. En Vázquez, I. (Coord.), Investigaciones multidisciplinares en Género, II Congreso Universitario (Nacional) Investigación y Género (pp. 871-886). https://idus.us.es/handle/11441/40469

Rebollo-Catalán, A., Mayor-Buzón, V. \& Vico-Bosch, A. (2018). La otra cara de la luna: la violencia invisible contra las mujeres en las redes sociales. En Donoso-Vázquez, T. \& Rebollo-Catalán, A. (Coords.). Violencias de género en entornos virtuales (pp. 105-120). Octaedro. 
Rebollo-Catalán, A. \& Mayor-Buzón, V. (2020). Adolescent Bystanders Witnessing Cyber Violence Against Women and Girls: What They Observe and How They Respond. Violence Against Women, 26(15-16), 2024-2040. https://doi.org/10.1177/1077801219888025

Reynolds, F., \& Shepherd, C. (2011). Young women's accounts of intimate partner violence during adolescence and subsequent recovery processes: An interpretative phenomenological analysis. Psychology and Psychotherapy: Theory, Research and Practice, 84(3), 314-334. https://doi.org/10.1111/j.2044-8341.2010.02001.x

Roca, N. \& Masip, J. (2011). Intervención grupal en Violencia Sexista: Experiencia, investigación y evaluación. Herder.

Ruiz-Pérez, I., Rodríguez-Madrid, N., Plazaola-Castaño, J., Montero-Piñar, I., EscribàAgüir, V., Márquez-Herrera, N., Sanz-Peregrín, C. \& Nevot-Cordero, A. (2013). Inhibiting and Facilitating Factors to End a Violent Relationship: Patterns of Behavior Among Women In Spain. Violence and Victims, 28(5), 884-898. https://doi. org/10.1891/0886-6708.VV-D-12-00055

Ruiz-Repullo, C. (2016). Voces tras los datos: una mirada cualitativa a la violencia de género en adolescentes. Instituto Andaluz de la Mujer. https://bit.ly/37UQs2j

Sabina, C. \& Ho, S.Y. (2014). Campus and college victim responses to sexual assault and dating violence: Disclosure, service utilization, and service provision. Trauma, Violence $\mathcal{E}$ Abuse, 15(3), 201-226. https://doi.org/10.1177\%2F1524838014521322

Santos, A., Matos, M. \& Machado, A. (2017). Effectiveness of a Group Intervention Program for Female Victims of Intimate Partner Violence. Small Group Research, 48(1), 34-61. https://doi.org/10.1177/1046496416675226

Sinko, L. \& Saint-Arnault, D. (2020). Finding the Strength to Heal: Understanding Recovery After Gender-Based Violence. Violence Against Women, 26(12-13), 1616-1635. https://doi.org/10.1177/1077801219885185

Storer, H.L \& Strohl, K.R. (2017). A Primer for Preventing Teen Dating Violence? The Representation of Teen Dating Violence in Young Adult Literature and Its Implications for Prevention. Violence Against Women, 23(14), 1730-1751. https://doi. org/10.1177/1077801216666725

Strauss, A., \& Corbin, J. (1998). Basics of qualitative research: Procedures and techniques for developing grounded theory. Sage Publications.

Taylor, S., Calkins, C.A., Xia, Y. \& Dalla, R.L. (2021). Adolescent Perceptions of Dating Violence: A Qualitative Study. Journal of Interpersonal Violence, 36(1-2), 448-468. https:// doi.org/10.1177/0886260517726969

Woodiwiss, J., Smith, K. \& Lokwood, K. (2017). Feminist narrative research: opportunities and challenges. Palgrave MacMillan.

Zapor, H., Wolford-Clevenger, C. \& Johnson, D.M. (2018). The Association Between Social Support and Stages of Change in Survivors of Intimate Partner Violence. Journal of Interpersonal Violence, 33(7), 1051-1070. https://doi.org/10.1177/0886260515614282

Fecha de recepción: 12 de enero de 2021.

Fecha de revisión: 10 de febrero de 2021.

Fecha de aceptación: 2 de mayo de 2021. 